\title{
Quantification of Fairness Bias in Relation to Decisions Using a Relativistic Fairness-Equity Model
}

\author{
Nicoladie D. Tam \\ Department of Biological Sciences \\ University of North Texas, Denton, TX 76023, USA \\ nicoladie.tam@unt.edu
}

\begin{abstract}
This study quantifies the fairness bias in relation to decision by a stimulusresponse function using a relativistic fairness-equity model. The interrelationship between fairness and decision is quantified by using an Ultimatum Game (UG) experimental paradigm in human subjects. The results showed that the fairness perception is shifted upward (toward a higher positive fairness baseline in the $y$-intercept of the stimulus-response function) for acceptance trials, without changing the slope (which corresponds to the fairness sensitivity). On the other hand, the fairness perception is shifted downward (toward a negative fairness baseline in the $y$-intercept) for the rejection trials. The analysis also showed that the fairness crossover point is shifted to the left for the acceptance trials, while the fairness crossover point is shifted to the right for the rejection trials. The analysis also showed that there is a singularity point, in which the most equitable offer (even-split) is always considered as the fairest, even when they rejected the offers. This absolute equity is rated as the fairest (even fairer than any of the hyper-equitable offers) independent of whether the subjects decided to accept or reject the offers. The changes in fairness perception are quantified by the shifting of the stimulusresponse curve up/down (changing the fairness baseline) or left/right (changing the fairness leniency), without changing the slope (the fairness sensitivity), when the decision is made to accept or reject the offers.
\end{abstract}

Keywords: Fairness bias; equity; egalitarianism, monetary gain; ultimatum game; decision.

\section{INTRODUCTION}

Fairness is one of the many social interactions that has been studied extensively to understand how it affects behavior [1-12]. It is also affects behavior in economic transaction [13-16], and distributive justice [17-19]. Fairness is one of the high-level cognitive concepts used not only in humans, but also in primates [20] to compare and contrast the differences in how we treat others, and how we want to be treated by others. Thus, it is important to determine how fairness is computed, so that the biases in fairness perception can be quantified, especially in relation to decision, i.e., how fairness bias can affect decision in social interactions.

\section{Fairness-Equity Models}

\section{LITERATURE REVIEW}

Most of the computational models of fairness [21-29] are based on the measure of equity. Most of these models are derived based on the economic game theories in monetary gain [15-18, 30], especially the inequity aversion model [16] to avoid unfairness or using mutual-max optimization [15] to achieve equity. These models may account for economic transaction, but 
they may not adequately account for the behaviors in social interactions. It is because social interactions are not always driven by maximizing personal gains, but the comparison between self and others with self-regarding and other-regarding concerns (when humans do not live in isolation by themselves, but live with the interactions with others). The inclusion of otherregarding concerns can change the dynamics of interactions and can affect the perception of fairness when the frame of reference in the comparison is switched, as proposed in our relativistic fairness-equity model [31-33].

\section{Relativistic Fairness-Equity Model}

The companion paper [33] has introduced the relativistic fairness-equity model to compare the difference based on a self-centered or other-centered frame of reference. By taking into the account the relative difference between self and others, it can account for the relativistic difference between what is fair for one person is unfair for another, when the frame of reference is switched from self to others. For instance, what is unfair to me is hyper-fair to you, if fairness is assessed by switching the frame of reference - from a self-centered frame of reference to an other-centered frame of reference. If the comparison is inclusive of both parties, then a global frame of reference is used instead of a local frame of reference, which also alters the assessment of fairness. Thus, fairness can be biased by a frame of reference used in computing the disparity between the parties. Such change in frame of reference can affect the perception of fairness, which can also affect the decision to accept or reject the social interactions.

\section{Quantification of Fairness relative to Decision}

As described in the companion paper [33], fairness perception can be quantified by a stimulusresponse function. The relativistic fairness-equity model can now be used to address the relationship between fairness and decision quantitatively, by describing how fairness perception is biased by the shifting of the stimulus-response curve relative to the selected frame of reference [32]. More specifically, if the perception of fairness is changed from unfair to fair by switching the frame of reference, then the decision to accept or reject an offer could be changed also. The ability to quantify fairness based on the stimulus-response function allows us to determine exactly how fairness is biased in making these decisions. In order to validate this hypothesis, we use the classic Ultimatum Game [13, 22, 26, 34-36] to determine the factors that may contribute to these biases, and how they can affect the social dynamics.

\section{Ultimatum Game Experimental Paradigm}

Ultimatum Game (UG) is one of the most widely used experimental paradigms to assessing the relationship between fairness and decision in behavioral sciences [13, 22, 26, 34-36]. It is a cognitive task used to determine the factors affecting the decision-making process, which can be dependent on how the human subject perceives whether the offer is fair or not. The task is a game in which a sum of money (such as \$10) is divided between two parties. The proposer offers a part of the money (such as $\$ 2$ ) to the responder, while keeping the remaining for himself/herself (such as \$8). The responder is given the choice to accept or reject the offer. The rule is that if the responder accepts the offer, both parties keep the money. If the responder rejects, both parties lose the money.

Numerous studies had shown that the decision to accept or reject an offer in UG is often dependent on the fairness perception [5, 8-12, 37-39]. The neurobiological substrates involved in fairness that affects decision are also well documented [2-4, 6, 7, 40]. Not only do humans reject unfair offers, monkeys also reject unfair offers [20]. This shows that the 
observable behavioral outcomes of unfair treatments are conserved across different species of higher animals.

\section{Objectives}

The objective of this study is to quantify the relationship between fairness and decision using the UG experimental paradigm, so that we can assess the interrelationship between fairness and decision in social interactions. Specifically, we will address the hypothesis that the decision to accept or reject a fair/unfair offer is logically consistent with how fairness is biased based on the relative frame of reference rather than some predefined social norms. To test the above hypotheses of fairness bias, we use stimulus-response function to determine how fairness bias (if exists) is shifted/skewed relative to the equity measure graphically.

\section{Experimental Paradigm}

\section{METHODS}

Healthy human volunteers were recruited to participate in this UG study. The human subjects were asked to play as the responders in UG to decide whether to accept or reject, while the computer played as the proposer to present the monetary offer to the responders on the computer screen. The computer offer was used as the proposer, since it is known that the perception of a human proposer vs. a computer proposer could bias the fairness perception of the subjects in UG $[8,37]$. We did not use any deception or confederates to alter the subject's fairness perception, so there were no experimental manipulations of the subject's fairness perception. The experiments were done by the subjects without any time pressure.

Nine randomized offers were proposed in the experiment - ranging from $\$ 1$ to $\$ 9$, without repeating the same offer (i.e., one-shot trial). The offer-ratios ranged from $\$ 1$ : $\$ 9$ (stingy offer) to $\$ 9: \$ 1$ (generous offer), which were presented in random orders. After the responder accepted or rejected the offer, the subject was asked to rate how fair the offer was to them (in a scale of +5 to -5 Likert scale [41]). The subject was also asked to rate other attributes (including how important fairness is; how important money is; how important winning is; whether they won the trial; in addition to other emotions: happy, angry, sad and jealous using the same rating scale). The list of attributes served, in part, as distracters to reduce the likelihood of skewing their responses to what they assume the experimenter expects from them.

The same pseudo-random sequence of monetary offers was used for all subjects by design, so that the experimental conditions were uniform across all subjects to ensure consistency. In order to quantify the fairness biases, we specifically measured the subject's self-reported ratings by design, because they represented the subject's own perception, filtered through their own subjective biases. By using the self-reported rating of fairness, if bias existed in their fairness perception, that bias would be reported as a skewed response, so the bias could be revealed and uncovered in the analysis. The study was approved by the University Institutional Review Board (IRB), and informed consent was provided prior to the beginning of the experiment.

\section{RESULTS}

Data were collected from 425 human subjects (age ranging from 18 to 80, median $=21$; mean $=$ 22.3; SD = 4.7; 275 female, 150 male). Figs. 1 and 2 show the self-reported fairness ratings of all the subjects with respect to the offer-ratios (sorted from the original randomized offerratios). Fig. 1 shows the stimulus-response function of the acceptance trials, while Fig. 2 shows the stimulus-response function of the rejection trials. 
Note that the same subject could accept one offer (such as $\$ 8: \$ 2$ ), while rejecting another offer (such as $\$ 3: \$ 7$ ). Thus, Fig. 1 includes all the acceptance responses for a specific offerratio; and Fig. 2 includes all the rejection responses that a subject rejected. This means that the responses of the same subject can be represented in both Figs. 1 and 2, depending on whether the decision is to accept or reject a particular offer. Therefore, the stimulus-response functions in Figs. 1 and 2 are representative of the overall generalized population, rather than the individual response of any specific person.

\section{Fairness Perception in Acceptance Decision}

Fig. 1 shows the fairness stimulus-response graph of the acceptance trials. The regression line $(r=0.967 ; r 2=0.935)$ shows a proportionality relationship between the fairness rating and the offer-ratios. This suggests that the perception of fairness is proportional to the equity-ratio between the two parties. The fairness crossover threshold is at offer-ratio $=\$ 2.5: \$ 7.5$. That is, it takes a minimum of a $\$ 2.5$ offer for the subject to consider the offer as neutrally fair. An offer below $\$ 2.5$ is considered unfair, and a proposal above $\$ 2.5$ is considered fair, as long as the subject accepts the offer.

Note that, interestingly, even though we never offered specifically the offer-ratio of $\$ 2.5: \$ 7.5$ in the experiment, the stimulus-response function revealed the exact crossover point for fairness. (We either offered $\$ 2: \$ 8$ or $\$ 3: \$ 7$ only.) This shows that quantifying the fairness perception by the stimulus-response graph allows us to determine the exact fairness crossover point for the human subjects to consider the offer as fair.

Most importantly, the stimulus-response graph shows a singularity point at offer-ratio $=1$. The fairness rating at offer-ratio $=\$ 5: \$ 5$ is the highest. In fact, it is twice as fair (fairness rating = 3.7) as the extreme hyper-fair/hyper-equitable $\$ 9: \$ 1$ offer (fairness rating = 1.7). The fairness rating for the absolute equity offer could have been rated at a fairness rating of 0.8 , if the regression line is used to interpolate the data. Thus, it reveals two interesting phenomena:

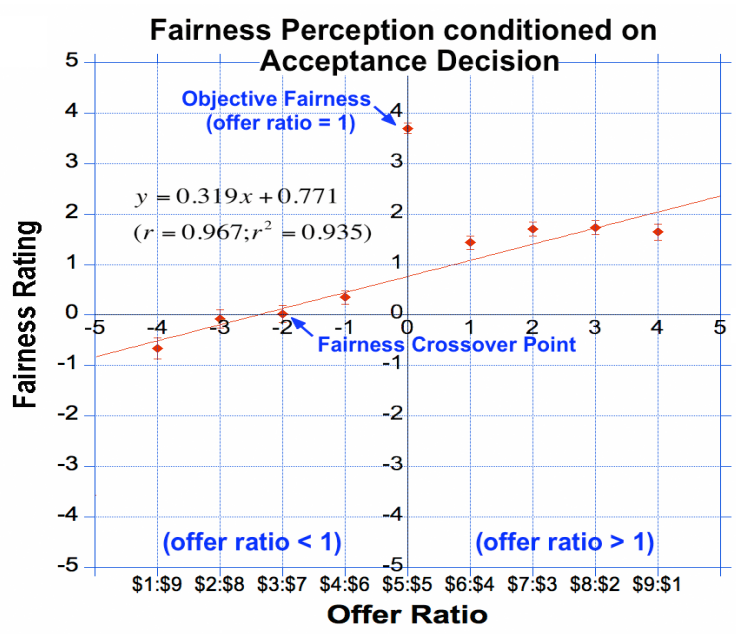

Figure 1:: Stimulus-Response Graph of Fairness vs. OfferRatios for Acceptance Trials. Curve-fitting is done by regression for all data points (excluding singularity-point at offer-ratio $=0$ ). It shows a linear proportionality relationship between fairness rating and offer-ratio. Note that the fairness rating at absolute equity (offer-ratio $=1$ ) is the highest, even higher than the hyper-fair offers (offer-ratio $>1$ ). The error bar represents standard error of mean (SEM).

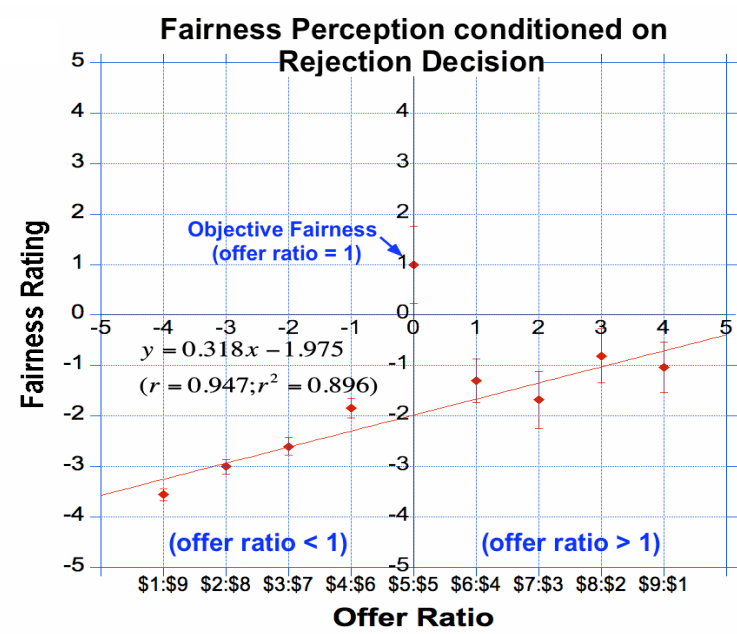

Figure 2: Stimulus-Response Graph of Fairness vs. Offer-Ratios for Rejection Trials. Curve-fitting is done by regression for all data points (excluding singularity-point at offer-ratio $=0$ ). It also shows a linear proportionality relationship between fairness rating and offer-ratio. Note that the fairness rating at absolute equity (offer-ratio $=1$ ) is still the highest, even when the human subject rejected the offer. It is even higher than the hyper-fair offers (offer-ratio > 1). 
First, the subject reported that the fairest offer is at absolute equity ( $\$ 5: \$ 5)$. They consider this equitable offer to be more fair than any other hyper-equitable/hyper-generous offers. That is, equality between two parties is considered more fair than any hyper-generous offers. This is counter-intuitive if the equity is the only measure used to determine fairness, because absolute equity (offer-ratio $=0$ ) is more fair than hyper-equitable/hyper-generous offers (offer-ratios > 1).

To account for this singularity point at absolute equity, the relativistic fairness-equity model captures this phenomenon by switching from the self-centered frame of reference in evaluating fairness to the other-centered frame of reference. If the self-centered frame of reference is used, the interpolation of the data points in the regression line suggests that the human subjects could have rated the fairness rating at 0.8 for the offer-ratio of $\$ 5: \$ 5$. Instead, the subjects actually rated it at 3.7, almost four times higher than predicted self-centered fairness rating. This suggests that the subject switched from the self-regarding concerns to the otherregarding concerns in social interactions.

When the self-centered frame of reference is used, what is hyper-equitable (offer-ratios $>1$ ) to one person is inequitable (offer-ratios $<1$ ) to the other person in comparison. Therefore, by switching the frame of reference in evaluating fairness, the dilemma of optimizing fairness for both can be resolved at absolute equity (offer-ratio $=1$ ). That is, when the offer is $\$ 5: \$ 5$, it is both equitable and fair for both parties. It is no longer fair for one person, but unfair for another person. This indicates that human subjects switched from a subjective self-centered frame of reference to an objective other-centered frame of reference that is inclusive of both self and others.

Second, the stimulus-response graph shows that the fairness crossover point is not centered at the axes-origin (offer-ratio $=\$ 5: \$ 5$ ), but at offer-ratio $=\$ 2.5: \$ 7.5$. This means that the fairness-equity curve is shifted to the left — to a more lenient consideration — to accept stingy offers, by taking into account the other-regarding concerns, when evaluating what is fair, and what is not fair.

The above two exceptions to the self-centered rule in fairness consideration indicate that the human subjects used both self-centered and other-centered frames of reference in assessing what is fair and what is not fair. The data strongly suggests that the human subjects did not simply use the equity measure - whether it is inequitable or hyper-equitable - as the only measure for fairness assessment. Instead, the subjects included the relativistic nature of fairness by considering both self-centered and other-centered frames of reference in the fairness assessment.

\section{Fairness Perception in Rejection Decision}

Fig. 2 shows the fairness stimulus-response graph for the rejection trials. The regression line $(r=0.947 ; r 2=0.896)$ also shows a proportionality relationship between the fairness rating and the offer-ratios, similar to the acceptance trials (see Fig. 1). The difference is that the fairness curve is shifted down relative to the acceptance curve. The y-intercept at the axesorigin (at offer-ratio $=1$ ) shifted down from the fairness rating of 0.8 (for acceptance trials in Fig. 1) to -2.0 (for rejection trials in Fig. 2). The fairness perception changed from a positive to a negative value. There is a $28 \%$ reduction in fairness perception (a 2.8 point reduction in a 10-point rating scale of fairness) for the decisions accepting the offers vs. the decisions rejecting the offers. The baseline fairness is shifted up to a more fair perception for the acceptance trials, while the baseline fairness is shifted down to an unfair perception for the rejection trails. This indicates the change in the baseline fairness level that is more fair if the 
human subjects accepted the offer vs. a change in the baseline fairness level that is more unfair if the subjects rejected the offer.

Note that the fairness crossover point is shifted to the right (Fig. 2) for the rejection trials, instead of being shifted to the left (Fig. 1) for the acceptance trials. The crossover point is shifted to the offer-ratio of beyond $\$ 9$ : $\$ 1$ (indicating greediness) for the rejection trials compared to the crossover point at offer-ratio of $\$ 2.5: \$ 7.5$ (indicating leniency). This indicates that for acceptance trials, the fairness stimulus-response curve is shifted to the left (to more leniency); while for rejection trials, the stimulus-response curve is shifted to the right (to more greediness). Thus, the stimulus response function revealed the change in fairness perception to become more lenient (crossover point shifts to the left) if the subjects accepted the offer vs. the change in fairness perception to being greedier (crossover point shifts to the right) when the subjects rejected the offer.

Note that the slope of the fairness stimulus-response function (which represents fairness sensitivity) remains rather constant for both decisions. The slope of 0.319 for the acceptance trials (regression line: $y=0.319 x+0.771$, see Fig. 1 ) is essentially the same as the slope of 0.318 for the rejection trials (regression line: $\mathrm{y}=0.318 \mathrm{x}-1.975$, see Fig. 2). The only difference between the fairness-equity curves in Figs. 1 and 2 is the y-intercept, representing a shifting of the curve down in fairness perception, without changing the fairness sensitivity (represented by the slope of the stimulus-response function).

This suggests that the decision to accept or reject an offer only affects the baseline level of fairness perception without affecting the fairness sensitivity. This shows that the relativistic fairness-equity model can account for the fairness biases quantitatively by the fairness stimulus-response function. It delineates not only the amount of bias, but also whether the bias is due to a change in sensitivity changes or a change in baseline.

\section{DISCUSSIONS}

The analysis shows that the fairness perception is related to the decision to accept or reject the offer. Quantification of fairness by the stimulus-response function shows that the perception of fairness changes with respect to the decision. Although the UG experiment does not differentiate whether fairness alters the decision or decision alters the fairness perception, it shows an interrelationship between fairness and decision. That is, the subjects changed their baseline fairness perception (represented by a shift in y-intercept) without changing the fairness sensitivity (represented by keeping the slope constant in the stimulus-response function) when the decision changed from acceptance to rejection. This delineates the specific fairness bias in relation to decision that is due to a change in baseline fairness level, but not fairness sensitivity. That is, the subjects responded with the same sensitivity to fair or unfair offers, but perceived the offers as more fair (higher baseline fairness level) when they decided to accept them than when they rejected them. Thus, the fairness bias related to decision lies in the change in baseline fairness perception rather than a change in the sensitivity.

The quantitative analysis of the stimulus-response function also reveals how the fairnessequity curve is shifted, depending on the decision. Specifically, when the fairness crossover point is shifted to the left, then the fairness bias is quantified as being lenient. When the fairness crossover point is shifted to the right, then the fairness bias is quantified as being greedy. At the same time, if the slope of the fairness-equity curve does not change, it means that there is no bias in the fairness sensitivity, i.e., the human subjects did not become more or 
less sensitive to fairness when they accepted or rejected to the offers, respectively. The only difference between the fairness perception related to the acceptance and rejection decisions is the shifting of the fairness-equity curve up or down, and left or right, without changing the slope.

The bias in fairness perception related to decision could possibly be related to the assumption or questioning of whether the responders were entitled to the money to start out with. That is, if the responders assumed that it is free money, then gaining any money would be more than fair, because they are not entitled to the money to start out with. But if the responders assumed that they have the rights to claim money (i.e., the money belongs to them) before the money was offered, then sharing that pot of money with the proposer who is not entitled to it would be unfair. Furthermore, if the responders assumed that neither one is entitled to the money to start out with, when the proposer offered to share the money between them, then it would be generous of the proposer to offer the money to them. As a response to the kindness, they would be lenient to the offer by considering slightly inequitable offer as fair (e.g., offerratio $=\$ 3: \$ 7$, by shifting the fairness crossover point to the left). This also can explain the shifting of the fairness crossover point to beyond $\$ 9: \$ 1$ (indicating greediness by shifting the fairness crossover point to the right), if the responders assumed that they are entitled to the money, but the proposer is not. Thus, this analysis shows that the fairness perception is not necessarily solely determined by the equity measure or by inequity aversion, but by the consideration of self-regarding and other-regarding concerns, whether they are entitled to the share that the proposer is offering.

This also suggests that the subjects did respond to the offer rationally without being irrational in their decision, when the difference in the response is the change in fairness bias. That is, their decisions are logically consistent with the fairness perception, without any logical inconsistency. It is only when equity/inequity is used as the sole measure to maximize selfgain, that it would be irrational if they throw away the money by rejecting the offers. This is in contrast to the assumptions that the rejection decisions are irrational in other UG studies [8, 42, 43].

The rejection decision may not necessarily be related to altruistic punishment, as other studies had shown that when the UG paradigm is modified slightly to use the rule in the Impunity Game (IG) $[39,44]$ that if the responder rejects the offer, only the responder loses the money while the proposer still keeps the money. In this case, the responders cannot possibly punish the proposer when they reject the offer; they can only punish themselves. Even under these conditions in the IG experiments, the responders still rejected the inequitable offers in IG, although the rejection rate is about half of that in UG [39]. The 50\%-chance rejection threshold for inequitable offers is similar to other reported rejection thresholds at below offer-ratio $=2$ : 8 for UG and IG; $[36,39,45]$. This suggests that their decision is more related to fairness than punishing the proposer. Altruistic punishment may happen to be an idiosyncratic side effect of the UG paradigm, when the responder rejects the unfair offer, because it is a forced-choice to punish the proposer when rejecting the offer. The UG game-rule does not allow for nonpunishing behavior on rejection. This results in a forced mutual-min (mutually destructive) outcome, if the responder rejects, and mutual-max (cooperative) outcome if the responder accepts, but nothing in between [15].

The neurobiological basis of fairness assessment and decision-making were identified [8, 43], which also support the neurological computation of fairness rather than an acquired behavior. The neural circuitries associated with fairness preference when accepting an equitable offer in UG are identified to be involved in the ventral striatum, amygdala, vmPFC (ventromedial 
prefrontal cortex), and orbitofrontal cortex (OFC) [3]. But the acceptance of inequitable offers is not associated with increased activity in these reward-related brain areas, but an increase in right VLPFC (ventral lateral prefrontal cortex) activation, and a decrease in insular cortex activity [3]. The rejection decision in UG is related to the vmPFC [43]. This suggests fairness is processed relatively automatically, and is innately driven by the reward circuitry, whereas unfairness aversion is driven more by a more cognitive-process via different neural circuitries. The fronto-striatal-thalamic network (consisting of the medial frontal gyrus, anterior insula, ventral striatum, and dorsomedial thalamus) is modulated by complex categorization uncertainty [46]. This neural circuitry may play a role in disambiguating the difference between equity and fairness and summaries the interrelationship between fairness and decision.

\section{CONCLUSION}

This study showed that fairness perception and fairness biases can be quantified by the stimulus-response function in relation to the decisions using the relativistic fairness-equity model. Using the UG paradigm, the interrelationship between fairness and decision is quantified by the fairness stimulus-response function. Specifically, fairness perception is biased toward a higher positive fairness baseline value when the subjects accept the offer compared to the rejection decision (by shifting the baseline of the fairness-equity curve, without changing the slope of the curve). Furthermore, the analysis also quantified that the fairness crossover point is shifted to the left or right according to the acceptance and rejection decision, respectively. This suggested that the decision to accept the offer is associated with leniency by considering inequitable offers as fair; while the decision to reject the offer is associated with greediness by considering hyper-equitable offers as unfair. The relativistic fairness-equity model can account for the singularity point that absolute equity is more fair than any hyper-equitable offers by including both self-centered and other-centered frames of reference in fairness assessment [33]. It also accounts for the shifting of the fairness-equity curves up/down and left/right according to the decision to accept or reject, relative to the selfcentered and other-centered frames of reference in the fairness considerations that include self-regarding and other-regarding concerns.

\section{ACKNOWLEDGEMENT}

I thank Ms. Krista Smith for providing helpful suggestions and proofreading the manuscript.

\section{REFERENCES}

1. Singer, T., H.D. Critchley, and K. Preuschoff, A common role of insula in feelings, empathy and uncertainty. Trends Cogn Sci, 2009. 13(8): p. 334-340.

2. Singer, T., et al., Empathic neural responses are modulated by the perceived fairness of others. Nature, 2006. 439(7075): p. 466-469.

3. Tabibnia, G., A.B. Satpute, and M.D. Lieberman, The sunny side of fairness: preference for fairness activates reward circuitry (and disregarding unfairness activates self-control circuitry). Psychol Sci, 2008. 19(4): p. 339-347.

4. Pillutla, M.M. and J.K. Murnighan, Unfairness, Anger, and Spite: Emotional Rejections of Ultimatum Offers. Org Behav Human Decis Proc, 1996. 68(3): p. 208-224.

5. Güroğlu, B., W. van den Bos, and E.A. Crone, Fairness considerations: increasing understanding of intentionality during adolescence. J Exp Child Psychol, 2009. 104(4): p. 398-409. 
6. Güroğlu, B., et al., Unfair? It depends: neural correlates of fairness in social context. Soc Cogn Affect Neurosci, 2010. 5(4): p. 414-423.

7. Seip, E.C., W.W. van Dijk, and M. Rotteveel, On hotheads and Dirty Harries: the primacy of anger in altruistic punishment. Ann N Y Acad Sci, 2009. 1167: p. 190-196.

8. Sanfey, A.G., et al., The neural basis of economic decision-making in the Ultimatum Game. Science, 2003. 300(5626): p. 1755-1758.

9. Rilling, J.K., B. King-Casas, and A.G. Sanfey, The neurobiology of social decision-making. Curr Opin Neurobiol, 2008. 18(2): p. 159-165.

10. Reuben, E. and F. van Winden, Fairness perceptions and prosocial emotions in the power to take. J Econ Psych, 2010. 31: p. 908-922.

11. Fehr, E. and S. Gächter, Altruistic punishment in humans. Nature, 2002. 415(6868): p. 137-140.

12. Takagishi, H., et al., Theory of mind enhances preference for fairness. J Exp Child Psychol, 2010. 105(1-2): p. 130-137.

13. Güth, W., R. Schmittberger, and B. Schwarze, An experimental analysis of ultimatum bargaining. J Econ Behav Organization, 1982. 3(4): p. 367-388.

14. Ochs, J. and A.E. Roth, An experimental study of sequential bargaining. Am Econ Review, 1989. 79(3): p. $355-384$.

15. Rabin, M., Incorporating fairness into game theory and economics. Am Econ Review, 1993. 83(5): p. 1281-1302.

16. Fehr, E. and K.M. Schmidt, A theory of fairness, competition, and cooperation. Quarterly J Econ, 1999. 114: p. 817-868.

17. Falk, A., E. Fehr, and U. Fuschbacher, On the nature of fair behavior. Econ Inquiry, 2003. 41(1): p. 20-26.

18. Konow, J., Which is the fairest one of all? A positive analysis of justice theories. J Econ Lit, 2003. 41: p. 1186-1239.

19. Rawls, J., A theory of justice1971, Cambridge: Harvard University Press.

20. Brosnan, S.F. and F.B. De Waal, Monkeys reject unequal pay. Nature, 2003. 425(6955): p. 297-299.

21. Page, K.M. and M.A. Nowak, A generalized adaptive dynamics framework can describe the evolutionary Ultimatum Game. J Theor Biol, 2001. 209(2): p. 173-179.

22. Braun, D.A., P.A. Ortega, and D.M. Wolpert, Nash equilibria in multi-agent motor interactions. PLoS Comput Biol, 2009. 5(8): p. e1000468.

23. Killingback, T. and E. Studer, Spatial Ultimatum Games, collaborations and the evolution of fairness. Proc Biol Sci, 2001. 268(1478): p. 1797-1801.

24. Nowak, M.A., K.M. Page, and K. Sigmund, Fairness versus reason in the ultimatum game. Science, 2000. 289(5485): p. 1773-1775.

25. Page, K.M., M.A. Nowak, and K. Sigmund, The spatial ultimatum game. Proc Biol Sci, 2000. 267(1458): p. 2177-2182.

26. Sigmund, K., C. Hauert, and M.A. Nowak, Reward and punishment. Proc Natl Acad Sci U S A, 2001. 98(19): p. 10757-10762.

27. Duan, W.Q. and H.E. Stanley, Fairness emergence from zero-intelligence agents. Phys Rev E Stat Nonlin Soft Matter Phys, 2010. 81(2 Pt 2): p. 026104. 
28. $\mathrm{Li}, \mathrm{X}$. and L. Cao, Largest Laplacian eigenvalue predicts the emergence of costly punishment in the evolutionary ultimatum game on networks. Phys Rev E Stat Nonlin Soft Matter Phys, 2009. 80(6 Pt 2): p. 066101.

29. Sánchez, A. and J.A. Cuesta, Altruism may arise from individual selection. J Theor Biol, 2005. 235(2): p. 233-240.

30. Bolton, G.E., A comparative model of bargaining: theory and evidence. Am Econ Rev, 1991. 81: p. 10961136.

31. Tam, D.N., Contributing factors in judgment of fairness by monetary value. BMC Neuroscience, 2011. 12(Suppl 1): p. P329.

32. Tam, D.N., Quantification of fairness bias by a Fairness-Equity Model. BMC Neuroscience, 2011. 12(Suppl 1): p. P327.

33. Tam, N.D., Quantification of fairness perception by including other-regarding concerns using a relativistic fairness-equity model. Advances in Social Sciences Research Journal, 2014. 1(4): p. 163-172.

34. von Neumann, J., O. Morgenstern, and A. Rubinstein, Theory of games and economic behavior1953, Princeton, NJ: Princeton University Press.

35. Camerer, C., Behavioral game theory: Experiments in strategic interaction2003: Princeton University Press.

36. Kagel, J.H. and A.E. Roth, The handbook of experimental economics1995: PRINCETON University Press.

37. Rilling, J.K., et al., The neural correlates of theory of mind within interpersonal interactions. Neuroimage, 2004. 22(4): p. 1694-1703.

38. Takagishi, H., et al., Neural correlates of the rejection of unfair offers in the impunity game. Neuro Endocrinol Lett, 2009. 30(4): p. 496-500.

39. Yamagishi, T., et al., The private rejection of unfair offers and emotional commitment. Proc Natl Acad Sci U S A, 2009. 106(28): p. 11520-11523.

40. Singer, T. and N. Steinbeis, Differential roles of fairness- and compassion-based motivations for cooperation, defection, and punishment. Ann N Y Acad Sci, 2009. 1167: p. 41-50.

41. Komorita, S.S., Attitude content, intensity, and the neutral point on a Likert scale. J Soc Psychol, 1963. 61: p. 327-334.

42. Civai, C., et al., Are irrational reactions to unfairness truly emotionally-driven? Dissociated behavioural and emotional responses in the Ultimatum Game task. Cognition, 2010. 114(1): p. 89-95.

43. Koenigs, M. and D. Tranel, Irrational economic decision-making after ventromedial prefrontal damage: evidence from the Ultimatum Game. J Neurosci, 2007. 27(4): p. 951-956.

44. Bolton, G.E. and R. Zwick, Anonymity versus punishment in ultimatum bargaining. Games Econ Behav, 1995. 10: p. 95-121.

45. Camerer, C. and R.H. Thaler, Anomalies: Ultimatums, dictators, and manner. J Econ Persp, 1995. 9: p. 209-219.

46. Grinband, J., J. Hirsch, and V.P. Ferrera, A neural representation of categorization uncertainty in the human brain. Neuron, 2006. 49(5): p. 757-763. 